\title{
The future SwissFEL facility - challenges from a radiation protection point of view
}

\author{
Claudia Strabel ${ }^{1, *}$, Albert Fuchs ${ }^{1}$, Roman Galev ${ }^{1}$, Eike Hohmann ${ }^{1}$, Roland Lüscher $^{1}$, Elisa Musto $^{1}$ and Sabine Mayer ${ }^{1}$ \\ ${ }^{1}$ Paul Scherrer Institute, CH-5232 Villigen, Switzerland
}

\begin{abstract}
The Swiss Free Electron Laser is a new large-scale facility currently under construction at the Paul Scherrer Institute. Accessible areas surrounding the $720 \mathrm{~m}$ long accelerator tunnel, together with the pulsed time structure of the primary beam, lead to new challenges for ensuring that the radiation level in these areas remains in compliance with the legal constraints. For this purpose an online survey system based on the monitoring of the ambient dose rate arising from neutrons inside of the accelerator tunnel and opportunely calibrated to indicate the total dose rate outside of the tunnel, will be installed. The presented study provides a conceptual overview of this system, its underlying assumptions and measurements so far performed to validate its concept.
\end{abstract}

\section{Introduction}

The Swiss Free Electron Laser (SwissFEL) [1] is the new large research facility at Paul Scherrer Institute (PSI), which will allow expanding the frontiers of knowledge in a wide range of scientific researches. It is foreseen to accelerate electrons up to an energy of $7 \mathrm{GeV}$ with a pulsed-time structure of the primary beam in the range of a few ps, and to produce FEL pulses covering the wavelength range of $1 \AA$ to $70 \AA$, i.e. $0.1-7 \mathrm{~nm}$. The accelerator will be operated with a maximum charge of $800 \mathrm{pC}$ per pulse and a maximum repetition rate of 100 $\mathrm{Hz}$. The main beam parameters are summarised in Table 1.

The final layout of SwissFEL is illustrated in Figure 1. It comprises an injector area (gun and booster), three linear accelerating sections, two parallel undulator lines (Aramis and Athos) and several experimental areas, for a total length of $720 \mathrm{~m}$. The facility is located underground, on the ground floor of a two-floor building, partly integrated in a regional recreation area (see Figure 2).
Table 1. Key beam parameters of SwissFEL.

\begin{tabular}{|c|c|}
\hline Beam parameter & Range \\
\hline Electron energy & $\max .7 \mathrm{GeV}$ \\
\hline Bunch length & $\sim \mathrm{ps}$ \\
\hline FEL wavelength & $1 \AA-70 \AA$ \\
\hline Charge per pulse & $800 \mathrm{pC}$ \\
\hline Repetition rate & $\max .100 \mathrm{~Hz}$ \\
\hline
\end{tabular}

The normal SwissFEL operation will give rise to secondary radiation fields deriving from the interactions of the primary beam with the beam line components; these radiation fields have been investigated [2] by means of Monte Carlo simulations. During normal

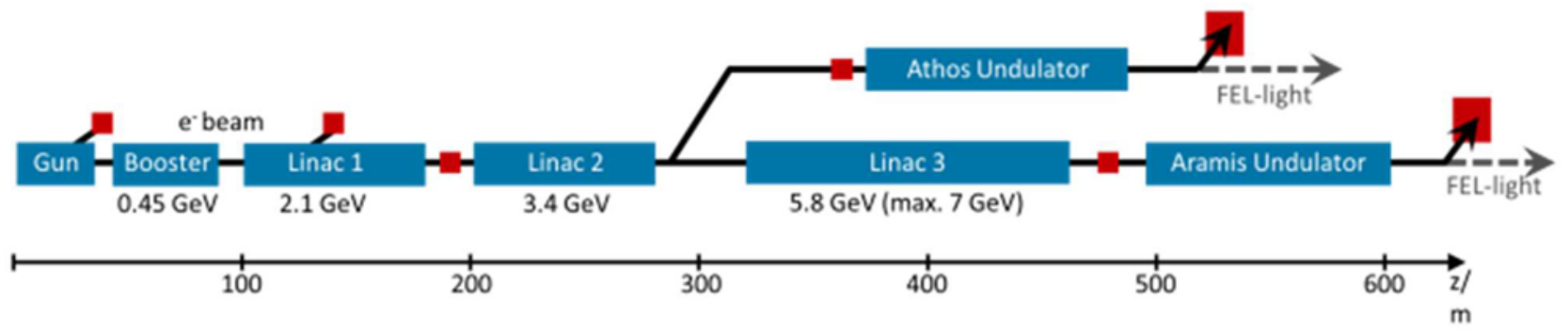

Fig. 1. The SwissFEL facility layout with the associated primary beam energies.

\footnotetext{
* Corresponding author: claudia.strabel $@$ psi.ch
}

(C) The Authors, published by EDP Sciences. This is an open access article distributed under the terms of the Creative Commons Attribution License 4.0 (http://creativecommons.org/licenses/by/4.0/). 


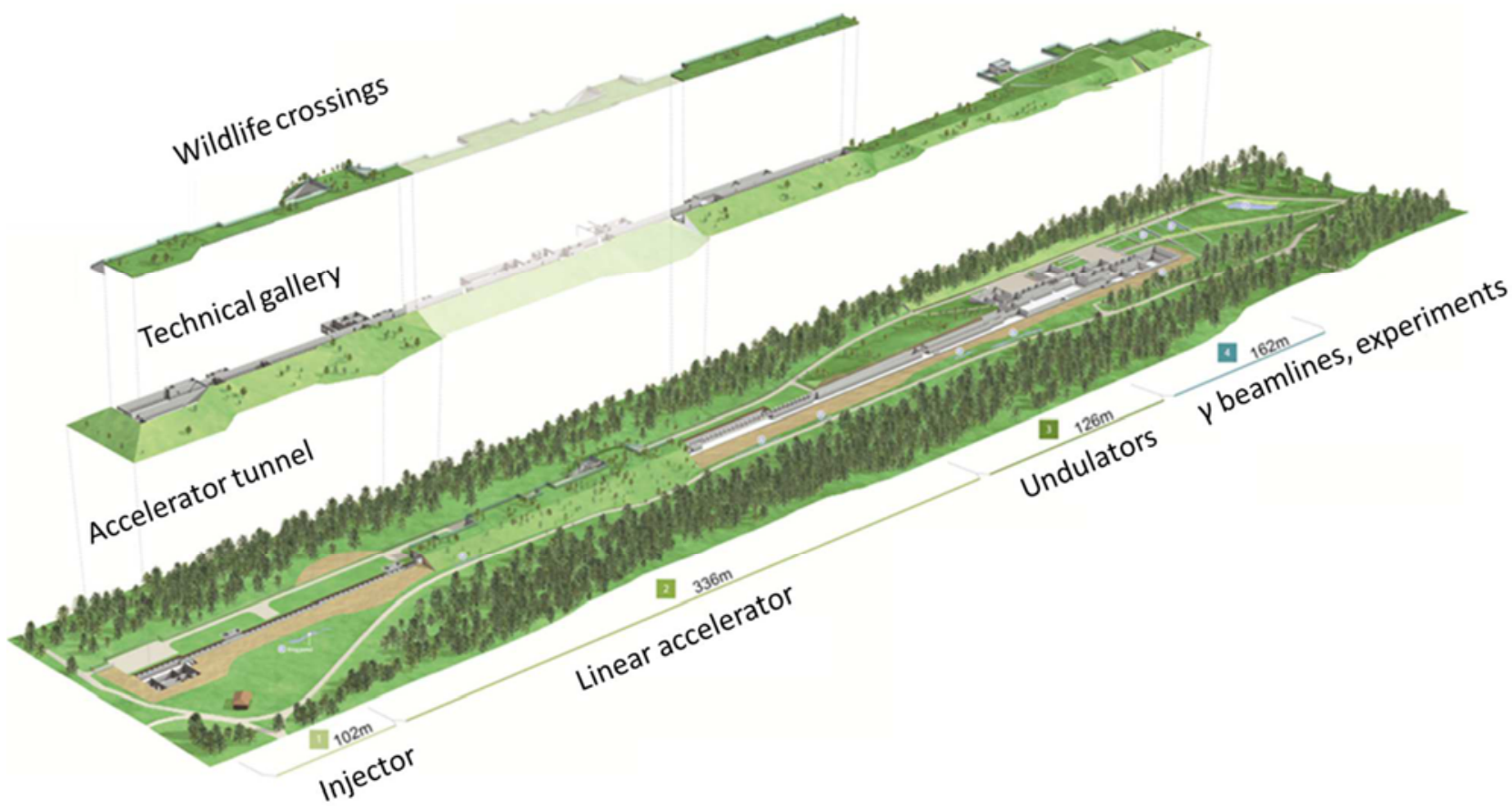

Fig. 2. The SwissFEL building site: With a total length of $720 \mathrm{~m}$, the accelerator, composed of the injector and linear accelerator, and the undulators are located in the lower floor of a two-storey building. Most of the building will be underground, but the upper floor (technical gallery) will only be semi-covered with soil, to ensure good maintenance access. Wildlife crossings above of the technical gallery allow wildlife to pass from one side of the facility to the other. The experimental area is a single-storey building, which is naturally covered by the surrounding land.

operation, the shielding of the accelerator vault, designed in the early stages of the project, can reduce the radiation in the accessible areas to an acceptable level in compliance with legal constraints. It can also cope with a major accident scenario of a full beam loss for less than a few seconds. The radiation level will routinely be monitored by passive devices distributed along the areas neighbouring the accelerator vault, accumulating for time periods of three months. However, since the guideline values according to Swiss radiological protection regulation [3] can be exceeded in shorter time periods, it is foreseen to install an online dose rate monitoring system called Dose Rate Protection System (DRPS). Its aim, concept, calibration and preliminary measurements will be explained in the following sections.

\section{The SwissFEL DRPS}

\subsection{Aim of the system}

The aim of the DPRS is to provide the SwissFEL facility with a system capable of monitoring the dose rate in the different accessible areas inside and outside of the facility. The system shall raise warnings in case that the indicated dose rate will approach the predefined limits, and shall provide a signal, which will lead to an immediate shut down of the beam in case that a violation of the guideline values occurs. Furthermore, the DRPS shall allow for a continuous monitoring of the radiation level in absence of the beam such that it can be ensured that all of the beam components possibly leading to radiation are switched off during maintenance work inside of the tunnel.

\subsection{Basic concept}

The dose rate expected in the various accessible areas inside and outside of the facility is low. Therefore the associated measurement uncertainties in the respective areas are large. A systematic and reliable monitoring of the dose rate outside of the SwissFEL accelerator tunnel is hence not possible. This became the major constraint to be taken into account for the development of the DRPS concept. Instead of measuring the dose rate in the accessible areas, the design of the DRPS has been based on measuring the dose rate inside the accelerator tunnel and on the assumption that it can be employed to extrapolate with a satisfactory accuracy the dose rate outside of the tunnel. This assumption is mainly based on the fact that the impact of a partial or a total beam loss is independent on the loss position of the beam but results in a comparable stray field.

The different magnets of the beam-line used for alignment and manipulation of the beam allow only small angular changes of the beam with respect to the nominal beam direction. In case of misalignment of the beam or an unsatisfactory beam alignment, electrons impinging on the beam pipe will predominantly produce Bremsstrahlung. The latter is mainly peaked in the forward direction such that it will interact with the 
magnets and other beam-line elements, which are distributed along the full length of the accelerator tunnel. It produces then secondary neutrons, photons and electrons. The major contribution to the total dose rate outside of the accelerator tunnel is expected from secondary neutrons. The DRPS will therefore employ extended range neutron REM counters (see Section 2.4) placed along the full accelerator length, which will measure the neutron dose rate inside of the tunnel. With an adequate calibration of the detectors the measured dose rate can be extrapolated towards the outside of the accelerator tunnel taking also the electromagnetic component into account. Figure 3 schematically illustrates the concept described above.

This assumption has been validated by means of simulations performed with the Monte Carlo particle transport code FLUKA [4, 5]. The simulations included an electron beam of variable energy impinging on a cylindrical copper target with a length of $30 \mathrm{~cm}$ and a radius of $10 \mathrm{~cm}$. These dimensions were chosen based on the assumption, that the beam can only be misaligned at small angles with respect to the beam direction and that the Bremsstrahlung arising from interactions with the beam pipe will be stopped when hitting a beam-line element. Finally, a simplified geometry with a concrete tunnel ceiling (thickness of $150 \mathrm{~cm}$ ) was used. The simulations showed that for beam energies lower than
$0.5 \mathrm{GeV}$, a full beam loss would lead to radiation levels outside of the accelerator tunnel that are in agreement with the guideline values, assuming a non-permanent stay (see Figure 4). For energies above $1 \mathrm{GeV}$ the expected dose rate arising from photons outside of the accelerator vault contributes by less than $7 \%$ to the total dose rate, which is mainly created by secondary neutrons. In fact, high energy Bremsstrahlung is mainly peaked in forward direction whereas the distance and shielding is minimal under an angle of $90^{\circ}$ with respect to the beam axis. In case of the latter, the spectral neutron distribution is almost independent of the energy of the primary electron beam for energies greater than 1 $\mathrm{GeV}$ (Figure 5, top) and the relative amount of neutrons with energies greater than $10 \mathrm{MeV}$ is less than $(1.3 \pm 7.2) \%$ close to the ceiling and $(60.4 \pm 0.3) \%$ outside of the accelerator vault (Figure 5 , bottom). As a result, the dose rate outside of the accelerator vault arises mainly from neutrons and can be measured inside with an adequate calibration.

Dedicated benchmark measurements of the DRPS concept will take place during the different commissioning phases of SwissFEL. Here, also gamma detectors will be employed in order to verify these numbers.

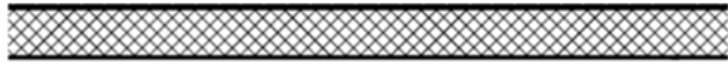

TECHNICAL GALLERY
$5.00 \mathrm{~m}$

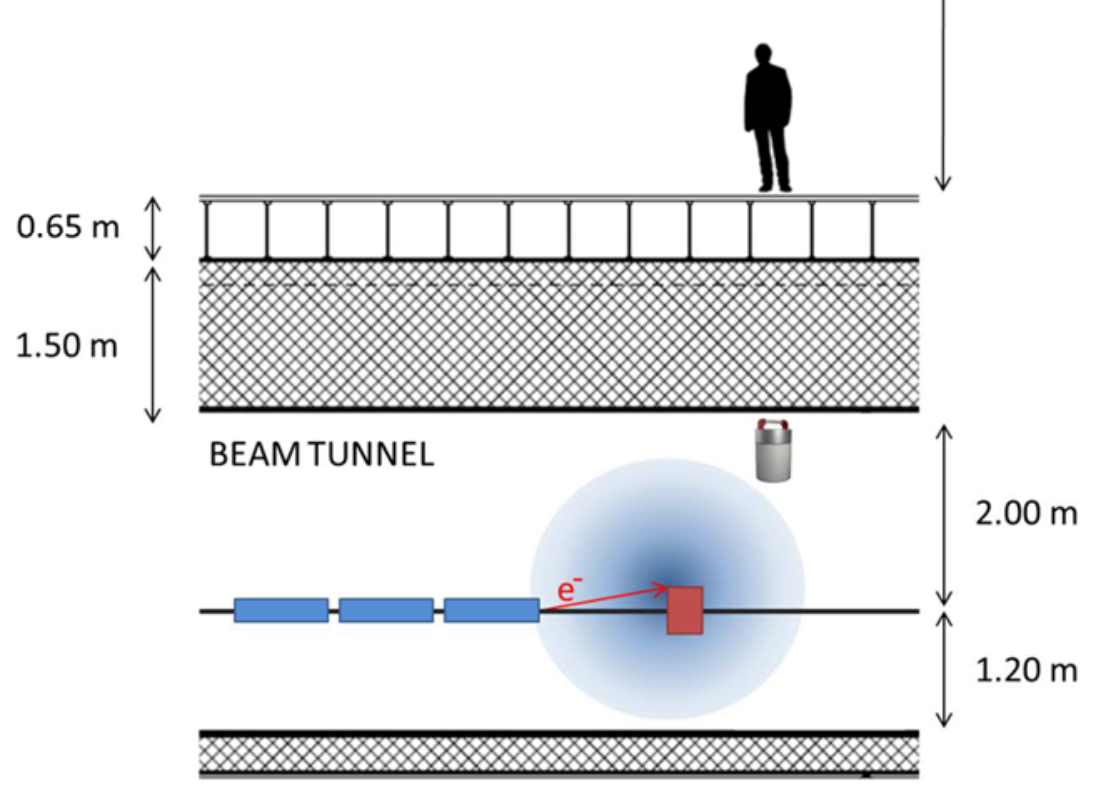

Fig. 3. Sketch of the DRPS concept. Misalignment of the beam results in Bremsstrahlung impinging on a magnet (presented by the red box). The total dose rate outside of the accelerator tunnel is monitored by a neutron detector, which is positioned inside of the accelerator tunnel and is calibrated such that it allows for the extrapolation of the total dose rate outside of the tunnel. See Section 2.4 for details about the LUPIN BF 3 -NP detector manufactured by ELSE Nuclear $[6,7,8]$ that is employed in the DRPS. 


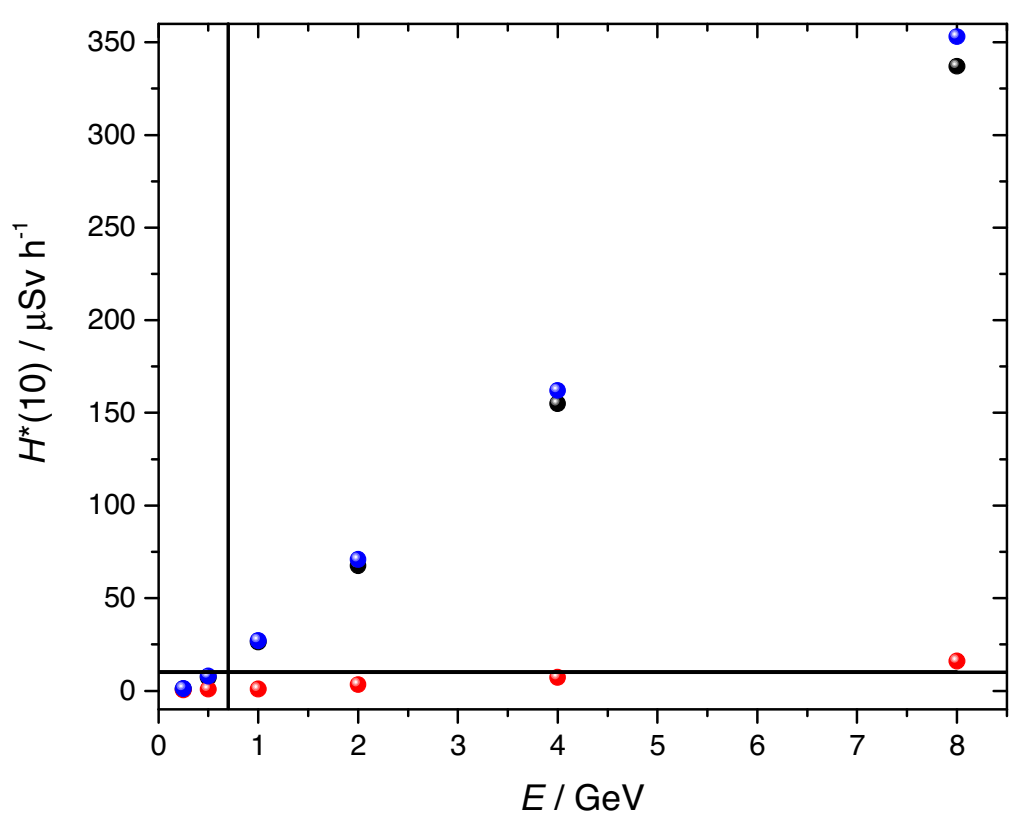

Fig. 4. Simulated dose rates outside of the accelerator vault, based on simplified assumptions and a full beam loss scenario arising from neutrons (black), photons (red) and total (blue). For electron energies less than $0.5 \mathrm{GeV}$ (vertical black line), the calculated dose rates are not exceeding $10 \mu \mathrm{Sv} / \mathrm{h}$.
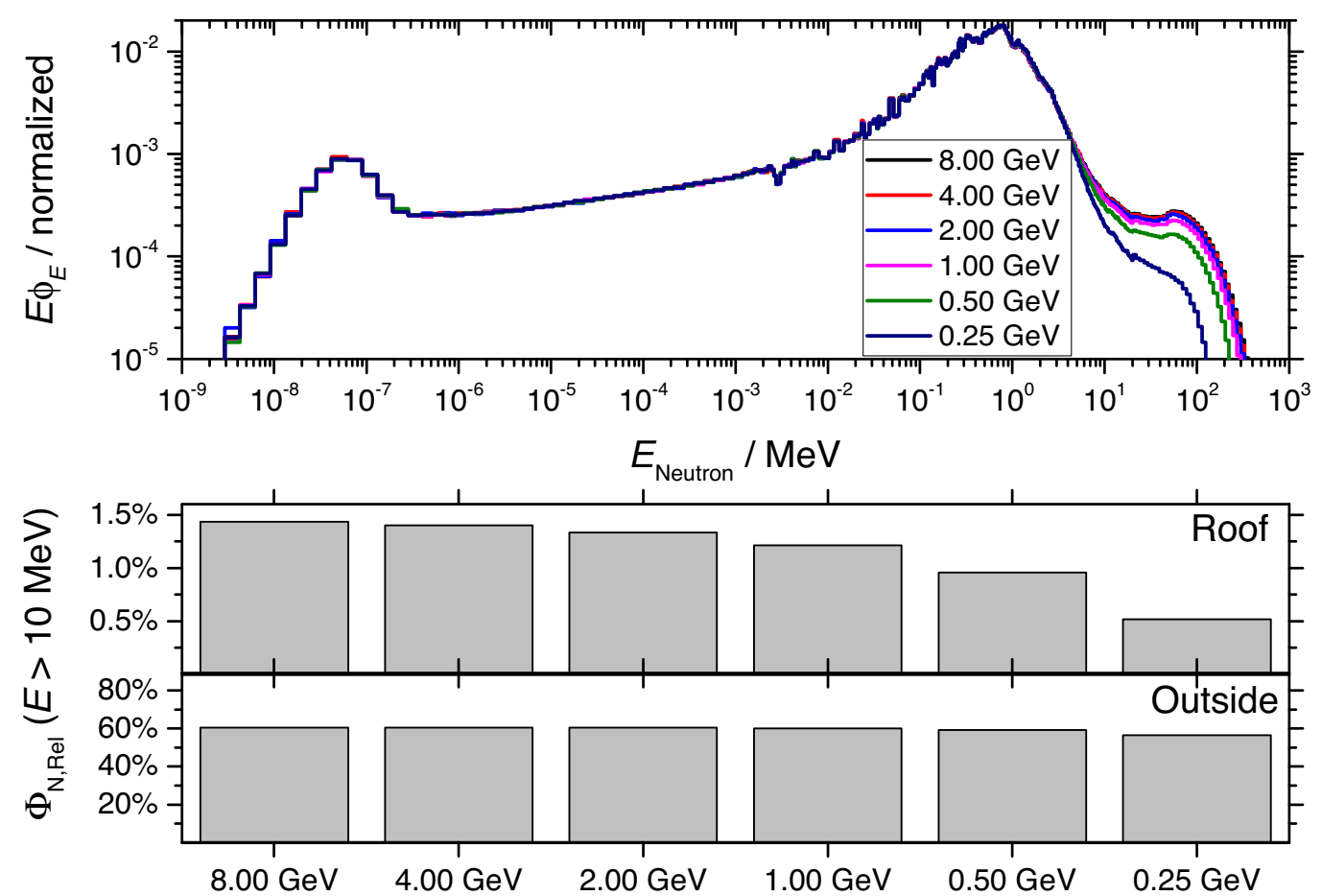

Fig. 5. Top: Simulated spectral neutron distribution near the roof of the SwissFEL vault for electrons impinging on a cylindrical copper target, for different electron energies. Bottom: Relative amount of neutron with energies greater than $10 \mathrm{MeV}$, for different electron energies close to the roof and outside of the accelerator vault. 


\subsection{Calibration procedure}

The DRPS processes the dose rate measured inside the tunnel and extrapolates it to evaluate the dose rate in the various accessible areas surrounding the tunnel. To do this with a satisfactory accuracy, an adequate calibration is needed. In order to do so, a two-step procedure is foreseen.

In a first step, the accelerator tunnel is divided into different classes according to its shielding configuration. These classes comprise for example ventilation and cooling shafts as well as beam line crossings, which all exhibit different reductions of the concrete shielding of the surrounding accelerator tunnel. For each one of these classes a basic calibration factor is calculated using Monte Carlo methods, using a detailed geometric model. Figure 6 shows an example of such a shielding class by depicting a typical cooling shaft, through which the usually $1.5 \mathrm{~m}$ thick concrete ceiling of the accelerator tunnel is significantly reduced towards the above-lying technical gallery. In case of overlapping classes, the most conservative class will be used.

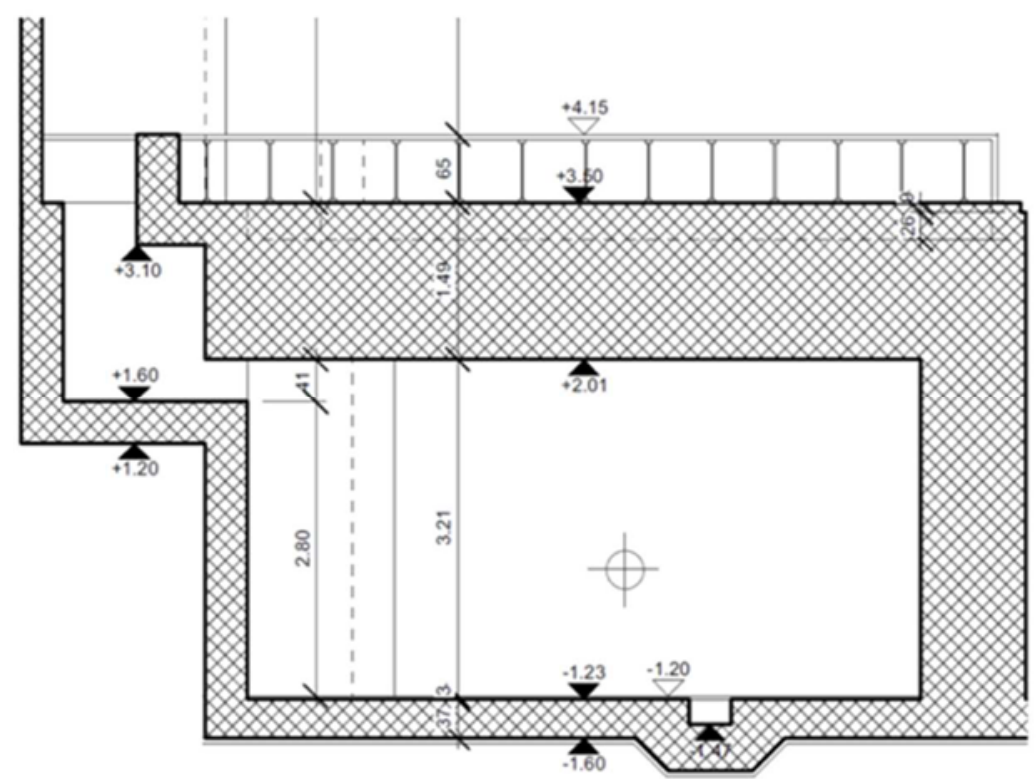

Fig. 6. Example of a calibration class with reduced shielding due to a cooling shaft with an opening of $41 \mathrm{~cm}$ from the accelerator tunnel towards the technical gallery above. The standard concrete shielding thickness towards the top gallery amounts to $1.5 \mathrm{~m}$.

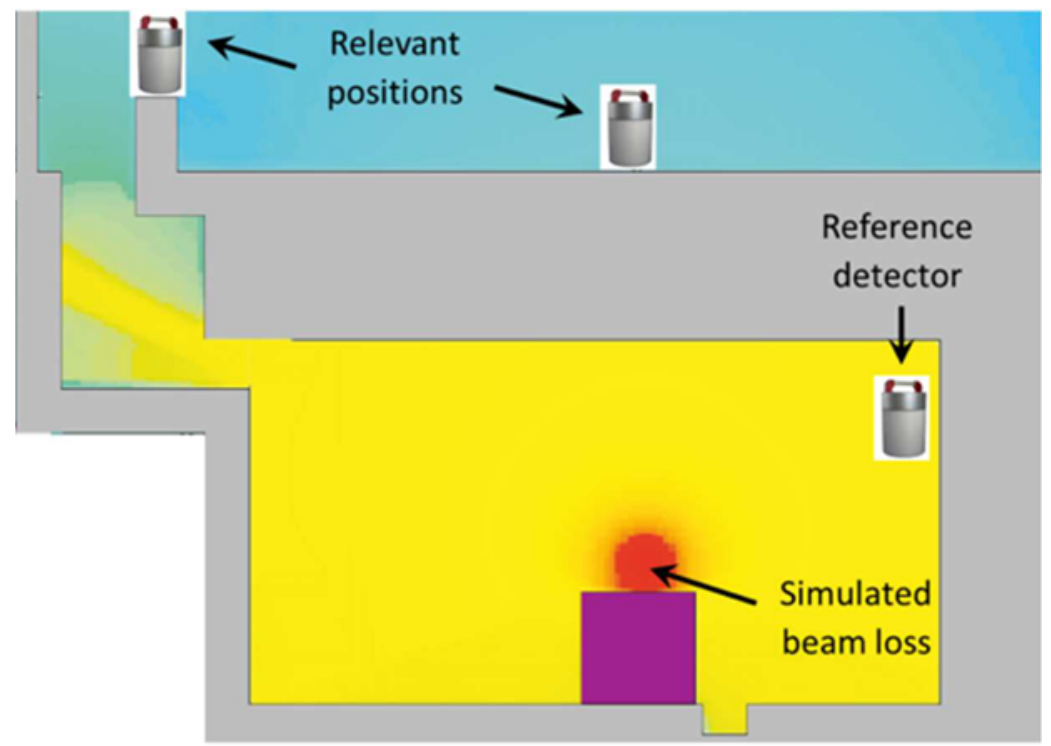

Fig. 7. Simulated dose rate generated from neutrons in case of a beam loss using FLUKA. The position of the reference detector inside of the accelerator tunnel is indicated on the right side. This detector will be used to extrapolate the neutron dose rate towards relevant positions in the accessible areas, such as the above-lying technical gallery. The extrapolation of the dose rate will be performed on the basis of the MC simulations and verified with dedicated benchmark measurements. 
In a second step, FLUKA will be used to simulate a beam loss for each type of shielding class. The results will then be utilised to check the difference between the neutron dose rate at the position of the DRPS monitoring device inside of the accelerator tunnel and the total dose rate including the electromagnetic component in the accessible areas of the facility and its surrounding. This is illustrated in Figure 7. The difference between the neutron dose rate inside and the total dose rate outside of the tunnel will then determine the calibration factor.

To guarantee the respect of the dose rate limits even if the beam loss doesn't occur directly above a particular detector, a threshold according to a worst-case scenario will be applied. Since this option significantly reduces the threshold and therewith may interfere with the SwissFEL operation, the signal coming from the different detectors will be simultaneously acquired and opportunely combined.

Given the anisotropy of the secondary radiation field along the beamline due to the non-homogeneous shielding, the configuration in terms of interspace between the detector elements needs to be investigated, optimized and validated.

The correct calibration of the various detectors will finally be validated with dedicated measurement campaigns in which independent neutron detectors using a different method of data evaluation as well as gamma detectors will be deployed.

\subsection{Preliminary measurements}

Performing measurements inside of the SwissFEL tunnel is a challenge for the employed neutron survey instrumentation particularly due to the pulsed structure of the radiation and the large photon contribution.

The LUPIN (Long Interval, Ultra-wide dynamic, Pile-up free, Neutron rem-counter) $[6,7,8] 5401 \mathrm{BF}_{3}-$ NP detector manufactured by ELSE Nuclear was selected as main measurement device for the DRPS. It consists of a $\mathrm{BF}_{3}$ neutron proportional counter, which is inserted in a cylindrical polyethylene moderator with a lead layer. It uses a front-end electronics based on a logarithmic amplifier. Its method allows the detection of every single radiation burst and is therefore ideally suited for the radiation fields at SwissFEL.

Next to the pulsed radiation fields, the detectors also have to cope with large radio-frequency (RF) fields, since they will be positioned above the beamline in proximity of the RF cavities responsible for providing the beam acceleration. To verify that the dose reading of the DRPS monitors is not affected by the RF fields, measurements with the LUPIN detector as well as other commercial available devices (LB6411 and WENDI-II) were performed at the test facility, where the SwissFEL RF cavities were commissioned. These measurements showed that there are no significant effects on the stability of the dose rate indication of the detectors due to the RF cavities.

\section{Summary}

The new large-scale facility SwissFEL currently under construction at PSI leads to challenges from a radiation protection point of view. An online survey system, the DRPS, will be used to monitor the ambient dose and ensure that the legal limits are respected. In this context, a brief overview of the system's concept, components and calibration has been provided.

\section{References}

1. R. Ganter (editor), SwissFEL CDR, http://www.psi.ch/swissfel/ (2012)

2. E. Hohmann, S. Reiche, A. Fuchs, R. Ganter, F. Le Pimpec, R. Lüscher, S. Mayer, T. Schietinger, PNST 4, 262-266 (2014)

3. Strahlenschutzverordnung, Der Schweizerische Bundesrat (2014)

4. T.T. Böhlen, F. Cerutti, M.P.W. Chin, A. Fassò, A. Ferrari, P.G. Ortega, A. Mairani, P.R. Sala, G. Smirnov and V. Vlachoudis, Nuclear Data Sheets 120, 211-214 (2014)

5. A. Ferrari, P.R. Sala, A. Fasso', and J. Ranft, CERN-2005-10 (2005), INFN/TC_05/11, SLAC-R773

6. M. Ferrarini, V. Varoli, A. Favalli, M. Caresana, B. Pedersen, Nucl. Instr. Meth. Phys. Res. Sec. A 613, 272 (2010)

7. M. Caresana, M. Ferrarini, G.P. Manessi, M.Silari, V. Varoli, Nucl. Instr. Meth. Phys. Res. Sec. A 712, 15 (2013)

8. M. Caresana, A. Denker, A. Esposito, M. Ferrarini, N. Golnik, E. Hohmann, A. Leuschner, M. LuszikBhadra, G. Manessi, S. Mayer, K. Ott, J. Röhrich, M. Silari, F. Trompier, M. Volnhals, M. Wielunski, Nucl. Instr. Meth. Phys. Res. Sec. A 737, 203 (2014) 